\title{
Warum eine Neuedition des Codex Madrid I von Leonardo da Vinci?
}

\author{
von Dietrich Lohrmann*
}

\begin{abstract}
Als 1968 die deutsche Ausgabe des bekannten, immer noch anregenden $\mathrm{Bu}-$ ches über die Ingenieure der Renaissance von Bertrand Gille erschien, erhielt der Nimbus Leonardos als eines bedeutenden, weit in die Zukunft weisenden Pioniers des Maschinenbaus zunächst einen schweren Schlag. Gille zeigte nämlich, dass viele von Leonardos Skizzen zur Mechanik thematisch nichts grundsätzlich Neues bieten, er vielmehr in einer langen Folge von Renaissance-Ingenieuren steht und er vieles von seinen Vorgängern in seine Skizzenbücher übernommen hat. ${ }^{1}$ Wie weit Leonardo seine Vorgaben bereichert, abgewandelt, verfeinert, verbessert hat, blieb vorerst offen. Etwa zur gleichen Zeit wurde aus Madrid bzw. aus Kalifornien bekannt, dass schon 1965 in der spanischen Nationalbibliothek zwei lang gesuchte Handschriften Leonardos endlich wieder aufgetaucht seien und der erste Band (künftig als Codex Madrid I bezeichnet) fast ausschließlich Ausarbeitungen zur Mechanik enthalte. Überhaupt sei es die umfangreichste Ausarbeitung des Autors zu den Maschinenelementen und ein wichtiger Beitrag zur Theorie.
\end{abstract}

Der designierte Herausgeber dieser Handschriften, der italo-amerikanische Ingenieur Ladislao Reti, hatte seine ersten Eindrücke schon im Sommer 1967 auf Einladung von Ludwig Heydenreich und Friedrich Klemm in München vorgestellt und noch im gleichen Jahr waren sie in der Technikgeschichte auch in deutscher Sprache erschienen. ${ }^{2}$ Aber das Buch von Bertrand Gilles war französisch in Paris schon 1964 herausgekommen und nur die deutsche Übersetzung hatte sich bis 1968 hingezogen. Reti widersprach der kritischen Einschätzung von Gille. Er betonte anhand des neuen Materials, Leonardo sei eben doch ein sehr origineller und weit in die Zukunft weisender Mechaniker gewesen. Gespannt wartete man deshalb auf die angekündigte Publikation des Fundes, der das definitiv beweisen sollte.

Die Edition Retis erschien 1974. ${ }^{3}$ Wir behandeln hier kurz ihre Entstehungsgeschichte und Struktur, fragen aber auch, ob mit dieser zweifellos

* Gerhard Dohrn-van Rossum danke ich für freundliche Beratung, Ulrich Alertz für Unterstützung bei der Gestaltung des Anhangs.

1 Bertrand Gille, Ingenieure der Renaissance, Wien u. Düsseldorf 1968.

2 Ladislao Reti, Die wiederaufgefundenen Leonardo-Manuskripte der Biblioteca Nacional in Madrid, in: Technikgeschichte 34, 1967, S. 193-225, hier S. 193; englische Fassung: ders., The Leonardo da Vinci Codices in the Biblioteca Nacional of Madrid, in: Technology and Culture 8, 1967, S. 437-445. Zur Person Retis vgl a. weiter unten Anm. 8.

3 Leonardo da Vinci, Codices Madrid, 5 Bde., Frankfurt a.M. 1974. Im Folgenden zit. als Reti 1974. 
sehr verdienstvollen Ausgabe bereits das Instrument vorliegt, das die Forschung benötigt, um auf Dauer weitere Fortschritte im Verständnis des Inhalts zu ermöglichen. In dieser knappen Begründung der letzthin negativen Antwort auf die Frage geht es nicht um eine ausführliche Darlegung der internationalen Forschungen zur Mechanik Leonardos aus den letzten fünfzig Jahren. Ziel ist nur eine Markierung der wichtigsten Etappen und Veröffentlichungen, angefangen vom Hauptereignis des Jahres 1965, der Wiederauffindung. Vorzustellen ist insbesondere die 1974 erschienene FaksimileEdition des Codex Madrid I in dessen heutiger Form, samt der Transkription durch Ladislao Reti, und andererseits die Anlage des Codex selbst in seinen wichtigsten Eigenheiten. Diese Eigenheiten beleuchten die Arbeitsweise und Intention Leonardos teilweise in einer ganz anderen Weise, als es die gedruckte Transkription des Faksimiles erkennen lässt. Um Leonardos Absichten gerecht zu werden und um vor allem die Bedürfnisse der Benutzer zu befriedigen und ihnen Hilfestellungen zu bieten, die sie in der eher ,asketischen“, teuren und nur schwer erhältlichen Faksimileausgabe von 1974 nicht finden können, ist somit eine neue Arbeitsausgabe des Codex Madrid 1 notwendig. Welche Ziele mit einer solchen Neuedition verfolgt werden, soll am Ende der folgenden Ausführungen durch einen Strukturvergleich der vorhandenen und der geplanten Edition anhand eines Modellkapitels illustriert werden.

\section{Die Wiederauffindung}

Die Überlieferung war für den Nachlass Leonardos bekanntlich nicht günstig. Eine große Zahl von Handschriften aus seinem Nachlass gelangte 1517 zwar testamentarisch in die Hände eines treuen Schülers, des Mailänders Francesco Melzi, doch begann spätestens nach dessen Tod um 1570 die „tragische Zerstreuung". Sie führte dazu, dass heute die wertvollsten Manuskripte in Mailand, Turin, Venedig, London, Windsor Castle, Paris und Madrid zu suchen sind und dass nach wie vor eine nicht näher zu präzisierende Menge von Bänden verschollen ist. ${ }^{4}$ Auch große Bibliotheken besitzen die so genannte Edizione nazionale der Handschriften und Zeichnungen Leonardos zumeist nur unvollständig. In diesem gewaltigen Editionsunternehmen des Verlags Giunti, Florenz, werden seit 1965 sämtliche bekannte Handschriften des groBen Florentiners als qualitativ hochwertige Faksimilia zusammengeführt und Umschriften beigefügt. Die Auflage ist weltweit begrenzt auf jeweils 998 Exemplare, ihr Preis aber so gewaltig, dass selbst die größten Bibliotheken, wenn

4 Den neuesten Überblick über die wichtigsten Handschriften Leonardos bietet Simona Cremante, Leonardo da Vinci, Florenz u. Mailand 2005, S. 351-635. Codex Madrid I (ms. 8937 der Madrider Nationalbibliothek) ist dort auf S. 578-591 mit elf Abbildungen vertreten. Ausführlicher äußert sich Augusto Marinoni, Leonardos Schriften, in: Ladislao Reti (Hg.), Leonardo. Künstler, Forscher, Magier, Frankfurt 1974, S. 56-85; auch in Carlo Zammattio, Augusto Marinoni u. Anna M. Brizio (Hg.), Leonardo der Forscher, Darmstadt 1981, S. 68-123. 
überhaupt, die insgesamt 50 Bände nur in Auswahl besitzen. Sie können diese auch nur unter besonderen Schutzbedingungen dem Leser zugänglich machen. ${ }^{5}$

Obwohl alle erhaltenen Werke inzwischen aus der typischen Spiegelschrift Leonardos in Normalschrift transkribiert sind, behindern schon diese äußeren Editionsumstände eine Erforschung und Auswertung durch einen weiteren Leserkreis als den der engeren Leonardo-Spezialisten. Nur für die wenigsten Forscher, deren Interessen sich auf Fragen richten, die über Leonardo hinausgehen, dürften die Bände, die sie benötigen, ohne erheblichen Aufwand erreichbar sein. Hinzu kommt, dass Leonardo seine Gedanken zu bestimmten Themen an den verschiedensten Stellen seiner Handschriften niederschrieb und der Benutzer diese nur bei Zugriff auf mehrere Bände zugleich in ihrer Bedeutung erfassen kann. Auch die internationale Leonardo-Forschung ist fast unüberblickbar geworden. Der gründliche Forscher muss sich ein näheres Eingehen auf die Untersuchung einer Spezialfrage bei Leonardo deshalb nicht selten untersagen. Eine benutzerfreundlichere Arbeitsausgabe des Codex Madrid I, des geschlossensten Werkes zur Mechanik, wäre deshalb alles andere als überflüssig.

Ein besonders glücklicher Fund zur Geschichte der Mechanik gelang, wie schon angedeutet, Anfang 1965 in der Nationalbibliothek Madrid. Zwei zuvor schon mehrfach bezeugte Codices Leonardos waren dort seit über 130 Jahren verschollen. ${ }^{6}$ Ein amerikanischer Hispanist, auf der Suche nach spanischen Heldenliedern, erhielt angeblich die Bände auf den Tisch, als er auf Verdacht zwei Leernummern des neuen Katalogs bestellte. Doch um mehr als eine gezielt verbreitete, oft wiederholte Legende handelt es sich dabei nicht. Die Leitung der Bibliothek, die lange vergeblich nach den verschollenen Bänden hatte suchen lassen und sie schließlich, um 100 Signaturnummern verwechselt, wiederfand, rief 1967 die besten Spezialisten der Leonardo-Forschung zusammen. Schnell stellte sich heraus, dass vor allem der stärkere der beiden Bände für die Geschichte der Mechanik von außerordentlicher Bedeutung sein würde.?

5 Zum Angebot des Verlags Giunti s. http://www.giunti.it/ [Stand: 1.4.2009], Preisliste auf Anfrage. Die meisten Bibliotheken sichern die Faksimileausgabe im Panzerschrank ihrer Rara und Rarissima. Die Bibliothek des Deutschen Museums, München, besitzt die zwölf Bände des Codex Atlanticus in der Ausgabe von Marinoni, die beiden Codices Madrid in der fünfbändigen Ausgabe Retis von 1974, die beiden Bände des Codex Arundel, ed. Pedretti, und den Codex Hammer bzw. Leicester mit Leonardos Studien zum Wasser. Sie verfügt nicht über die zwölf Pariser Notizbücher.

6 Erster Band mit der Aufschrift (18. Jh.) „Tratado de Estatica y Mechanica en Italiano“, dazu Hinweis von anderer Hand (17. Jh.) auf „Leonardo da Vinci pintor famoso“. Zweiter Band mit der Aufschrift „Tratados varios de Fortificacion, Estatica y Geometria escritos en Italiano, por los Anos de 1491 como se vé à la vuelta del fol. 157. Advertiendo que la Letra de este Libro està al reves".

7 Abweichend von der Version Retis von 1967 (wie Anm. 2) betont die Einleitung zur Faksimileausgabe (Reti 1974, Bd. 3, S. 13) den wesentlichen Anstoß zur Wiederauffindung durch den französischen Leonardo-Forscher André Corbeau. Nach dessen Darstellung erfolgte die Wiederauffindung zwischen dem 23. und 29.4.1965. Corbeau hatte seit 1957 
Den Auftrag zur Herausgabe erhielt mit Ladislao Reti einer der erfahrensten Kenner spätmittelalterlicher und frühneuzeitlicher Mechaniktraktate. Der 1901 in Fiume (Veneto) geborene Forscher, ausgebildet noch am Polytechnikum in Wien zum Chemieingenieur, danach in Bologna auch als Geisteswissenschaftler, war spätestens seit den 1950er Jahren mit grundlegenden Arbeiten über Renaissance-Ingenieure wie Leonardo, Francesco di Giorgio Martini und Juanelo Turriano hervorgetreten, zudem auch philologisch ausgewiesen und so für die Veröffentlichung der neu aufgefundenen Handschriften in Madrid der ideale Herausgeber. Noch im Mai 1966 anlässlich einer Tagung in Los Angeles hatte er offenbar von der bedeutenden Wiederentdeckung in Madrid keine Kenntnis und sprach, angeregt durch André Corbeau, nur von bisher vergeblicher, aber eventuell aussichtsreicher Suche. ${ }^{8}$ Erst Anfang 1967 benachrichtigte ihn (nach Angabe von Bern Dibner) die Spanische Nationalbibliothek. Nun gingen die Dinge schnell voran. Reti nahm umgehend ein Flugzeug nach Madrid, überzeugte sich von der Authentizität der Handschriften und unterrichtete schon im Februar die amerikanische Presse, die u.a. in der New York Times ausführlich berichtete. ${ }^{9}$

Bereits Anfang Juli 1967 folgte sein wissenschaftlicher Vortrag im Zentralinstitut für Kunstgeschichte in München, der noch im gleichen Jahr in Technology and Culture und der deutschen Zeitschrift Technikgeschichte erschien. $\mathrm{Zu}$ den beiden wiederentdeckten Handschriften liefert schon dieser Beitrag gründliche und eindringende Einzelangaben. Herkunft und Datierung der Handschrift sind weitgehend vorgeklärt, Abbildungen zeigen besonders bemerkenswerte Maschinenelemente wie Schraubenwinden, Rollen- und Kugellager, den Einsatz von Antifriktionsmetall, Zahnradgetriebe mit epizykloidalen Zähnen, aber auch bereits (ruhiger laufende) Riemenantriebe, eine Vorform der Pelton-Turbine, Studien zu Zahnrädern und Kettentrieben, die Regulierung einer Kreisbewegung durch schwere Pendel u.a.m. Ein wesentliches Problem wird von Reti gleich am Anfang herausgestellt: „Wir wissen noch nicht, ob Codex I das Buch über die Maschinenelemente ist, auf das sich Leonardo an anderen Stellen bezieht". ${ }^{10}$

Nachforschungen zu den Handschriften in Madrid unternommen, vgl. André Corbeau, La découverte des manuscrits de Léonard de Vinci à la Bibliothèque nationale de Madrid, 1969, 16 S. (Text für Leonardo-Konferenz 1968 in Florenz, eingesehen in Paris Bibliothèque nationale de France Imprimés), und ders., Les manuscrits de Léonard de Vinci, Caen 1968. Zur irrtümlichen alten Signatur (Aa 12 statt Aa 120) im so genannten Indice Goncalez der Madrider Nationalbibliothek vgl. Reti 1974, Bd. 3, S. 11.

8 Bern Dibner, Éloge Ladislao Reti 1901-1973, in: ISIS 65, 1974, S. 376ff.; auch in: Technikgeschichte 41, 1974, S. $73 \mathrm{ff}$.

9 Reti (wie Anm. 2), S. 193, Anm. 4.

10 Ebd., S. 196. 


\section{Retis Faksimile-Edition von 1974}

Retis Arbeit an der überaus schwierigen Transkription und Übersetzung der beiden Madrider Handschriften füllte die Jahre bis zu seinem Tod im Oktober 1973. Seit 1972 fand der Ingenieur Unterstützung durch Italiens besten Kenner der speziellen Paläographie und Philologie Leonardos, Augusto Marinoni. ${ }^{11}$ In vorbereitenden Studien untersuchte Reti u.a. das in älteren Ingenieurhandschriften kaum vertretene Material über Wellenlager und Getriebe. ${ }^{12}$ Dazu fand er überraschende Übereinstimmungen in dem bekannten Maschinenbuch des lombardischen Ingenieurs Augusto Ramelli (1588), dessen Quellen man bisher kaum kannte. ${ }^{13}$ Inhaltliche Abgleichungen mit anderen Handschriften Leonardos ergaben wichtige Ergänzungen. Nur ein kleiner Teil der Ergebnisse dieser vergleichenden Untersuchungen fanden Aufnahme in den schmalen dritten Band der 1974 erschienenen fünfbändigen Faksimileausgabe. Die Beschreibung der Handschrift Madrid I ist dort relativ kurz geraten, vermutlich wegen des frühen Todes Retis. Einzelerörterungen zur Mechanik sind in ihm nicht enthalten, auch keine genauere Inhaltsübersicht, wohl aber ein nützliches, wenn auch keineswegs vollständiges Wortregister

11 Im Titel der Ausgabe von 1974 ist die Mitwirkung von Marinoni nicht angegeben. Vgl. aber die Internetseite Marinonis, auf der von seiner Mitarbeit an der Transkription 19721975 berichtet wird (http://www.legnano.org/reteciv/marinoni/html/biografia.htm [Stand: 25.5.2009]) und die Copyrightseiten von Reti 1974, Bd. 4 Codex Madrid I: Transkription von Ladislao Reti, Übersetzung von Gustav Ineichen, Friedrich Klemm, Ludolf von Mackensen und Reinhild Richter, wo es auf der Rückseite des Titelblattes heißt: „Besonderer Dank gebührt Augusto Marinoni, der nach dem Tod von Ladislao Reti der abschließenden Arbeit an der Transkription seine wertvolle Hilfe lieh."

12 Ladislao Reti, Leonardo on Bearings and Gears, in: Scientific American 224, 1971, S. 101-110. Ansätze hierzu bereits bei Franz Maria Feldhaus, Technik der Vorzeit, Leipzig 1914, Sp. 599, der u.a. auf Antifriktionsrollen Leonardos 1496 im Pariser Ms. J verweist.

13 Ladislao Reti, Leonardo and Ramelli, in: Technology and Culture 13, 1972, S. 577-605. Weitere Vorstudien Retis waren u.a.: ders., Le arti chimiche di Leonardo da Vinci, in: La Chimica e l'Industria 34, 1952, S. 655-667 u. 721-743; ders., Leonardo da Vinci nella storia della macchina da vapore, in: Rivista di Ingegneria, 1956-1957, S. 27-38; ders., Tracce di progetti perduti di Filippo Brunelleschi nel Codice atlantico di Leonardo da Vinci, Lettura vinciana 4, Firenze 1965; ders., Leonardo da Vinci the Technologist. The Problem of the Prime Movers, in: International Leonardo Symposium University of Los Angeles, The Elmar Belt Library 1966; ders., Il moto dei proietti e del pendolo secondo Leonardo e Galileo, in: Le Machine 1, 1968, S. 63-89; ders. u. Bern Dibner, Leonardo da Vinci Technologist. Three Essays on Some Designs and Projects of the Florentine Master, in: Adapting Machinery and Technology to the Problems in Art, Industry and War, Norwalk, CT 1969; ders., The Problem of Prime Movers, in: Charles D. O’Malley (Hg.), Leonardo's Legacy. An International Symposium, Berkeley, Los Angeles 1969, S. 67-100; ders., The Double Acting Principle in East and West, in: Technology and Culture 11, 1970, S. 178-200; ders., Leonardo da Vinci and the Graphic Arts. The Early Invention of Relief Etching, in: Burlington Magazine 113, 1971, S. 189-195; ders., Leonardo da Vinci and Cesare Borgia, in: Viator 4, 1973, S. 333-368; ders., Leonardo and the Beginnings of Factories with a Central Source of Power, in: 450th Anniversary of the Death of LdV, Boston Studies Phil. Science 11, 1974, S. 4-115. Eine umfassende Bibliographie der Arbeiten Retis ist mir nicht bekannt. Die meisten Arbeiten besitzt das Istituto di storia della scienza in Florenz. 
von Marinoni (S. 127-148) und eine Auflistung der inhaltlichen Bezüge zwischen Madrid I und anderen Handschriften Leonardos, die der Benutzer berücksichtigen, von der Handschrift ausgehend aber jeweils mühsam suchen muss (Bd. 3, Anhang A). Die Edition selbst enthält keine Sachkommentare. Auch sind die Bezüge zwischen den Texttranskriptionen im Band 4 und den jeweils zugehörigen Zeichnungen im Band 1 nicht immer leicht herzustellen. Ausdrücklich erklärt Marinoni: „Es fehlen leider die Register und die Beschreibungen der Zeichnungen, die Herr Ladislao Reti noch erstellen wollte. "“14

Im Herbst 1973 beim Tode Retis war die Gesamtdisposition der Einleitung und der Transkriptionen einschließlich Übersetzungen so weit gediehen, dass das Gesamtwerk 1974 gleich in mehreren Sprachen erscheinen konnte. Die inhaltliche Auswertung verlagerte man in einen ebenfalls noch von Reti angeregten Sammelband. In ihm behandelten die seinerzeit besten Spezialisten folgende Einzelthemen: Leonardos Schriften im Gesamtüberblick (Marinoni), Leonardo als Festungsbaumeister (Heydenreich), Leonardo als Konstrukteur von Kriegsmaschinen (Dibner), seine Studien zu Hydraulik und Materialbelastung (Zammatio), sein Interesse für Uhrentechnik (Bedini und Reti) sowie seine mechanischen Studien insgesamt (Reti). Auch dieser wichtige Sammelband kam englisch, französisch, italienisch und deutsch noch im gleichen Jahr 1974 heraus und erfuhr 1980 bis 1981 in mehreren Sprachen eine dreibändige Neubearbeitung, die weit verbreitet werden konnte. ${ }^{15}$ Allerdings beschränken sich diese Untersuchungen in bewundernder Grundhaltung auf die besonders sensationellen, als zukunftsträchtig angesehenen Zeichnungen des Meisters. Eine Erschließung des Gesamtinhalts seiner neu erschlossenen Handschriften war nicht beabsichtigt und ist bis heute nicht verwirklicht. Vom Gesamtinhalt des Codex Madrid I dürfte kaum ein Zehntel des Inhalts näher untersucht sein. Reti bemerkt selbst, dass insbesondere im Bereich der Uhrentechnologie des Codex Madrid I noch Forschungsbedarf für Jahrzehnte bestehe.

Die nachfolgende Forschung hat das voraufgehend angedeutete Tempo nicht beibehalten können. Retis Edition und andere Veröffentlichungen galten als unbestrittene Meisterleistung. Sie wirkten zugleich aber auch als Hemmnis, denn die eindrucksvollsten und schönsten Zeichnungen waren nun schon aus dem Gesamtwerk herausgezogen und das enorme technische und philologische Wissen, das zur weiteren Auswertung notwendig erschien, war nur wenigen gegeben. Friedrich Klemm erläuterte 1975 zunächst die Bezüge zwischen Leonardo als Ingenieur und den Vorläufern des 15. Jahrhun-

14 Reti 1974, Bd. 3, S. 127.

15 Leonardo. Künstler, Forscher, Magier (wie Anm. 4). Der faszinierendste Beitrag dieses Bandes ist in mancher Hinsicht Silvio A. Bedini und L. Reti, Leonardo und die Uhr, S. 240-263. Er trägt wesentlich zum Verständnis einer Anzahl sonst schwer zu deutender Skizzen des Codex Madrid I bei, erscheint aber leider in der Neuauflage des Werks von 1981 nicht mehr: Leonardo der Künstler, der Forscher, der Erfinder, 3 Bde., Stuttgart u. Zürich 1981, und Sonderausgabe Wiss. Buchgesellschaft, Darmstadt 1981. 
derts. ${ }^{16}$ Drei Jahre später folgte sein Überblick über Physik und Technik bei Leonardo, hauptsächlich nach Anregungen Retis. ${ }^{17}$ Marco Cianchi lieferte 1984 einen knapp gefassten, nützlichen und gut illustrierten Überblick über die wichtigsten Bereiche von Leonardos Maschinenstudien, Kriegsmaschinen ebenso wie hydraulische Maschinen, Flugmaschinen und Mechanik. Ausgewählt sind Beispiele aus sämtlichen Handschriften des Meisters. ${ }^{18}$ Thematische Ordnung und Umsetzung in die Formelsprache der modernen Mechanik war 1989 das Anliegen des Wissenschaftsphilosophen Herbert Maschat, eine sehr hilfreiche Arbeit auch diese, nur dass sie die Vorläufer und Nachfolger Leonardos kaum einbezieht und somit den Genius weiter singularisiert. Leonardos starke Verbindung zur älteren Physik und Mechanik wie etwa zu der für ihn wichtigen Impetustheorie wird nicht erkennbar. ${ }^{19}$ Danach erlahmte die Leonardo-Forschung zur Mechanik in Deutschland, während in Italien das Istituto di storia della Scienza in Florenz unter der Leitung von Paolo Galluzzi eine Serie großer Ausstellungen in Gang brachte, die viel zur Kenntnis der frühen Renaissance-Ingenieure beitrugen, angefangen von Brunelleschi bis hin zu Leonardo. Kataloge von hoher Qualität ergänzten den Bau sorgfältig geplanter Maschinenmodelle. Ausstellungen gingen nach Frankreich, England, USA, Kanada, Israel, Japan, Österreich, nicht jedoch nach Deutschland. ${ }^{20}$

16 Friedrich Klemm, Leonardo da Vinci als Ingenieur und die Ingenieure seiner Zeit, in: ders., Zur Kulturgeschichte der Technik. Aufsätze und Vorträge, Deutsches Museum München 1979, S. 123-138 (zuerst 1975). Wichtig sind nach wie vor die Pionierarbeiten von Pierre Duhem vom Anfang des 20. Jahrhunderts zur Statik Leonardos und seiner Vorgänger (Les origines de la statique, 3 Bde., Paris 1905/06) sowie seine dreibändige Untersuchung der von Leonardo benutzten Autoren bzw. der Autoren, die ihn gelesen haben (Études sur Léonard de Vinci. Ceux qu'il a lus et ceux qui l'ont lu, 3 Bde., Paris 1906-13 [ND 1984]). Das Werk zur Statik erschien auch in englischer Übersetzung (The Origins of Statics. The Sources of Physical Theory, Dordrecht 1991).

17 Friedrich Klemm, Physik und Technik in Leonardo da Vincis Madrider Manuskripten, in: ders. (wie Anm. 16), S. 109-122 (zuerst 1978).

18 Marco Cianchi, Die Maschinen Leonardo da Vincis, Florenz 1984 (auch in italienischer, englischer und französischer Fassung erschienen und somit geeignet zum Vergleich der jeweiligen Fachterminologie).

19 Herbert Maschat, Leonardo da Vinci und die Technik der Renaissance, München 1989. Dazu Rezension von Eberhard Knobloch in: Technikgeschichte 57, 1990, S. 368-370. Zum Impetus bei Leonardo vgl. die anregenden Bemerkungen von Michael Wolff, Geschichte der Impetustheorie. Untersuchungen zum Ursprung der klassischen Mechanik, Frankfurt a.M. 1978, S. 266, 274 u.a.

20 Paolo Galluzzi (Hg.), Prima di Leonardo. Cultura delle macchine a Siena nel Rinascimento, Mailand 1991; ders., Les ingénieurs de la Renaissance. De Brunelleschi a Léonard de Vinci, Florenz 1995; ders., Mechanical Marvels. Invention in the Age of Leonardo da Vinci, Florenz 1997. Eine Ausstellung von in Polen gefertigten Maschinenmodellen folgte 2006 im Industrion Kerkrade (Niederlande), zeigte aber keinen Bezug zu den Manuskripten aus Madrid. Vgl. a. Marcus Popplow, Die Rückkehr des Künstler-Ingenieurs. Tendenzen und Perspektiven der Forschung zu Leonardo da Vinci, in: NTM. Zeitschrift für Geschichte der Naturwissenschaft, Technik und Medizin 16, 2008, S. 133-144, der ebenfalls eine deutliche Verlangsamung der Leonardo-Forschung im Bereich der Technik feststellt. 
Ein Mangel der neueren Forschungen zur Mechanik Leonardos, insbesondere im Codex Madrid I, liegt darin begründet, dass sie den großen Florentiner vornehmlich in Bezug zur Entwicklung von Physik, Mechanik und Technik des 17. bis 20. Jahrhunderts setzen und nicht das herausarbeiten, was er bereits vorfand. Wie er dies im Einzelnen verarbeitete, ob seine Nachfolger im 16. bis 17. Jahrhundert davon bereits Kenntnis erhielten und wie sie ihrerseits reagierten, kommt erst in neuesten Versuchen andeutungsweise zum Tragen. Zunächst prüfte man vor allem Leonardos Aussagen auf ihre Vereinbarkeit mit modernen Erkenntnissen und ließ dabei beiseite, was überholt schien. Andere rügten das Fehlen einer präzisen mechanischen Terminologie, die bekanntlich erst seit den Arbeiten eines Galilei, Stevin, Descartes und Newton entwickelt worden ist. Unberücksichtigt bleibt dabei, dass Leonardo als Autodidakt über eine beträchtliche Bibliothek verfügte (eine Liste im Codex Madrid II hat dies noch deutlicher werden lassen). Nachweislich hat er viel gelesen und gelegentlich auch seine Quellen verraten wie etwa Blasius Pelacani oder Albert von Sachsen, Autoren des 14. bis 15. Jahrhunderts. ${ }^{21}$

Unberücksichtigt blieb lange auch, dass seine Ausarbeitungen, obwohl überwiegend unveröffentlicht, im 16. Jahrhundert eine größere Wirkung ausgelöst haben, als das Schicksal seiner Handschriften es nahezulegen scheint. Schon am Beispiel seiner Traktate über Anatomie und Perspektive ergibt sich, dass Aufzeichnungen Leonardos im 16. Jahrhundert mehreren Gelehrten zugänglich waren. Sarlio, Cardano und Biondi verweisen auf sie. Anderes kann sich mündlich durch Leonardos Schülerkreis in Mailand verbreitet haben. Speziell seine Mechanik scheint erheblichen Einfluss auf Cardano, Ramelli und den Kommentar des Bernardino Baldi zur aristotelischen Mechanik ausgeübt zu haben (verfasst 1582, Erstausgabe Mainz 1621). ${ }^{22}$ Alfredo Serra bezweifelt in einer Studie über Kapitel 16 dieses Kommentars allerdings eine direkte Einwirkung in diesem Zusammenhang. ${ }^{23}$ Neue kinematische Ansätze erge-

21 Die Wirkung der Schriften des so genannten kleinen Albert (Albertucius) auf Leonardo wird untersucht von Duhem, Études (wie Anm. 16), Bd. 1, S. 14-50. Jürgen Sarnowsky, Die aristotelisch-scholastische Theorie der Bewegung. Studien zum Kommentar Alberts von Sachsen zur Physik des Aristoteles, Münster 1989, geht auf diese Fragen nicht ein, er untersucht die (fachlich begrenzte, aber pädagogisch beachtliche) Originalität des Albertucius innerhalb der Physikliteratur des 14. Jh. Arturo Uccelli, Leonardo da Vinci. I libri di meccanica nella ricostruzione di Arturo Uccelli, Mailand 1940, liefert einleitend S. XXXV-CLV einen nützlichen Überblick über die Leonardo bekannten älteren Werke. Genaue Verweise auf die einzelnen Texte Leonardos sind damit nicht verbunden. Madrid I war noch unbekannt.

22 Cardano: Zahlreiche verstreute Hinweise bereits bei Theodor Beck, Beiträge zur Geschichte des Maschinenbaues, Berlin 1899 und ders., in: Zeitschrift des Vereines deutscher Ingenieure 1906, S. 524-531, 562-569, 645-651, 777-784; Ramelli: vgl. Reti 1972 (wie Anm. 12); Baldi: vgl. Wolff (wie Anm. 19), S. 255ff. u. 282, Anm. 52.

23 Antono Becchi, Leonardo, Galilei e il caso Baldi, Venedig 2004; vgl. ders., Wusste Galileo davon? Die Architektur als Theatrum pro experimentali philosophia, in: Max-Planck multimedial. Jahrbuch 2007 (MPI für Wissenschaftsgeschichte, Berichte, Dokumente), 
ben sich schließlich aus Studien von Graham Hollister Short zur Zahnradtechnik (sector and chain), zu den Vorformen der Newcomenschen Dampfmaschine und zur Literatur über Leonardos Beitrag zu Textilmaschinen. ${ }^{24}$ All dieses müsste in einem neu zu erstellenden Kommentar Berücksichtigung finden.

\section{Die Struktur der Handschrift Codex Madrid I}

Die genannten Forschungen mögen dem Leser als so reichlich erscheinen, dass sie die Notwendigkeit einer Neuedition kaum erkennen lassen. Doch einerseits sind sie weit zerstreut, also bei Einzelrecherchen kaum mit Sicherheit aufzufinden, und zum anderen haben wir noch nicht die Handschrift selbst betrachtet. Diese Handschrift, Madrid Biblioteca Nacional 8937 (Madrid I), ist ein merkwürdiges Gebilde. Sie enthält die weitaus ausführlichsten, thematisch geschlossensten Untersuchungen Leonardos zur Mechanik, die bisher bekannt sind, und zerfällt, wie bereits Reti erkannte, in einen theoretischen und einen praktischen Teil. Der überwiegend theoretische Teil beginnt jedoch, anders als die Edition es zeigt, im heutigen Codex vom Ende her, der überwiegend praktische Teil vorne.

Für beide Teile bereitete Leonardo zunächst sechs Lagen mit jeweils acht Doppelblättern vor, zusammen 192 Blätter. Möglicherweise war es seine Absicht, diese in zwei Stapeln nebeneinander liegenden Lagen gleichzeitig bearbeiten zu können. Der theoretische Teil sollte den Anfang bilden, wenn wir Leonardos Aussage vertrauen, die Theorie habe voranzugehen, was jedoch vom Zusammenhang her nicht zwingend erscheint. ${ }^{25}$ Sicher ist: Der theoretische Teil trägt eine von hinten nach vorn laufende Zählung der Folien von 1 bis 95 in Leonardos charakteristischer Spiegelschrift, jeweils auf der Versoseite der heute gültigen modernen Zählung; zeichnerisch wirkt dieser Teil zunächst weniger ansehnlich. Die (heute) erste Hälfte des Bandes hat dagegen nur eine, wahrscheinlich die ursprüngliche Blattzählung. Dieser Teil des Bandes beeindruckt sofort durch die außerordentliche Sorgfalt und Plastizität der meisten Zeichnungen. Es sind Apparaturen, die einzeln mit ausführlicher Erläuterung eine ganze Seite füllen können (fol. $4^{\mathrm{r}}, 14^{\mathrm{v}}, 15^{\mathrm{r}}, 16^{\mathrm{r}}, 30^{\mathrm{r}} \mathrm{usw}$.). Öfter freilich bieten sie mehrere, vielfach sehr unterschiedliche und völlig heterogene Mechanismen auf derselben Seite, dazu nachgetragene Einzelstudien wie etwa auf fol. $7^{\mathrm{r}}$. Eindeutig handelt es sich um Beobachtungen aus der Praxis, so insbesondere bei den umfangreichen Studien zur Form und Funktionsweise der Zahnräder (fol. 5, 116).

Online: http://www.maxplanck.de/bilderBerichteDokumente/dokumentation/jahrbuch/ 2007/wissenschaftsgeschichte/forschungsSchwerpunkt/pdf.pdf [Stand: 29.4.2009]).

24 Romano Nanni u. Graham Hollister Short, Les machines proto-industrielles de Léonard, in: Journal de la Renaissance 5, 2007, S. 11-115.

25 Cod. Madrid I fol. 164 (d.h. im theoretischen Teil): „Questa si dimanda pratica, ma ricordati di mettere la teorica dinanzi.“ - „Dies erfordert Praxis, aber erinnere dich daran, die Theorie voranzustellen." 
Dieses Material zur praktischen Mechanik hat einen solchen Umfang angenommen, dass es bald auf Blätter des zweiten Teiles ausuferte und dort bis mindestens fol. $124^{\mathrm{v}}$ (ursprüngliche Zählung von hinten $67^{\mathrm{r}}$ ) vordrang. ${ }^{26}$ Andererseits erscheinen theoretische Ausführungen zu Statik und Gewichten auch im ersten Teil, so auf fol. 71 bis 79. Die Richtung von Leonardos Eintragungen innerhalb der beiden Teile scheint nach den Ausführungen einer neueren Untersuchung einer amerikanischen Romanistin gelegentlich zu wechseln. ${ }^{27}$ Leonardo war offensichtlich ein sehr kreativer, aber auch genialisch zerstreuter Autor. Die Besonderheiten sind vielfach ungeklärt, sie betreffen einen groBen Teil des Gesamtbands und sollten im Kommentar jeweils angezeigt werden.

Ergänzend zur rückläufigen Foliozählung zeigt auch die Zählung der meist kleinen Skizzen Leonardos im theoretischen Teil eine aus heutiger Sicht rückschreitende Richtung. Diese Skizzenzählung läuft auf den ursprünglichen Blättern $1^{\mathrm{r}}$ bis $20^{\mathrm{r}}$ von Nr. 1 bis Nr. 100. Sie erfasst aber längst nicht alle Skizzen (manche sind offenbar nachgetragen) und wechselt teilweise in der Richtung von oben nach unten, von unten nach oben bzw. von links nach rechts und rechts nach links. ${ }^{28}$ Eine zweite Skizzenzählung beginnt fol. $170^{\mathrm{r}}$ gleich mit 15 und reicht bis 90 auf fol. 158 r. Eine Zählung der Lagen durch Leonardo ist nicht erhalten. Die vorhandene könnte aus dem späteren 16. Jahrhundert stammen. Sie zieht sich durch den gesamten Band erneut von hinten nach vorn und ist beim Neueinband im 18. Jahrhundert nicht mehr beachtet worden, denn sonst hätte man damals den Band anders gestaltet.

Kein Zusammenhang besteht (trotz einiger zufälliger Übereinstimmungen) zwischen dieser Lagenzählung und den zahlreichen internen Verweisen Leonardos, die Johnston auf Lagen und Blätter des Codex Madrid I beziehen wollte. ${ }^{29}$ Reti kannte die alte Markierung, zählte die Lagen aber trotzdem im heutigen Sinn von vorn nach hinten. ${ }^{30}$ Insgesamt konnte er sich vor seinem Tode nicht mehr dazu durchringen, seine Edition entsprechend den Angaben des Autors zumindest im zweiten Teil „,on hinten“ anfangen zu lassen.

\section{Zusammenfassung}

Zusammenfassend lässt sich festhalten: Die Edition Retis von 1974 ist zweifellos eine gute Faksimileausgabe mit einer höchst wertvollen Transkription, einer guten, wenn auch teilweise leicht modernisierenden Übersetzung und einem knappen, nur allgemeine Informationen vermittelnden Kommentar-

26 Eigene Beobachtungen und Kommentar von Reti in: Reti 1974, Bd. 3, S. $32 \mathrm{ff}$.

27 Marina della Putta Johnston, Leonardo da Vinci's Codex Madrid I. The Creation of the Self as Author, Diss. University of Pennsylvania 2000, S. 28ff. u. 38 (Online: http:// repository.upenn.edu/dissertations/AAI9965465/ [Stand: 1.4.2009]). Die Wertung von Codex Madrid I als fast abgeschlossenem, systematisch angelegtem Werk durch die Autorin ist m.E. nicht zu halten.

28 Eine erste Zusammenstellung hierzu nach eigener Beobachtung liegt vor.

29 Johnston (wie Anm. 27), S. 47ff. Erste Überprüfungen stimmen skeptisch.

30 Reti 1974, Bd. 3, S. 26-29. 
band. Trotzdem entspricht sie nicht den vollen Absichten des Autors und vor allem nicht den Bedürfnissen des Benutzers, denn die Bände sind selten, relativ schwer zugänglich, und es fehlen vor allem die Beschreibungen der einzelnen, weit über tausend Zeichnungen, die als Maschinenelemente oder Gruppen solcher Elemente für die meisten Benutzer rätselhaft wirken. Da ihr Sinn und ihre Struktur aus dem ständig wechselnden Zusammenhang nicht ohne weiteres erschließbar sind, wäre es die erste und wichtigste Aufgabe einer Neuedition, hier Klarheit zu schaffen. ${ }^{31}$

Des Weiteren ist zu festzuhalten, dass wir mit der Faksimileausgabe von 1974, ohne inhaltliche Übersicht und Einzelkommentar, zwar den heutigen Zustand der Handschrift erkennen, nicht aber die ursprüngliche Abfolge. Wir erhalten auch keine allgemein verständliche Präsentation des Inhalts im Einzelnen und erst recht nicht, wie es letztlich doch notwendig wäre, die Vielfalt der oft wechselnden Ideen und ihre Stellung in der weiteren bzw. vorauf gegangenen Entwicklung der mechanischen Wissenschaft. ${ }^{32}$ Die Abschnitte zur Mechanik Leonardos, die sich in zahlreichen neueren Kunstbüchern finden, helfen hier nicht weiter. Sie bieten in der Regel einen sehr begrenzten Kanon besonders schöner und modern wirkender Zeichnungen. Nur mit sehr allgemeinen Kurztiteln versehen, vermitteln sie von der Intensität der Beobachtungen, Reflektionen und Ideen des Meisters wie gleichzeitig auch von den Schwächen seiner Präsentation keine ausreichende Vorstellung. ${ }^{33}$ Zum Schluss sollen

31 Die Neuedition ist zunächst als Internet-Edition geplant. Ein Antrag für die zur Durchführung nötigen Personalmittel ist seitens der Deutschen Forschungsgemeinschaft erfreulicherweise bewilligt worden. Für eine wirksame Anschubfinanzierung des Projekts danke ich der Ursula und Bernhard Plettner- Stiftung im Stifterverband für die Deutsche Wissenschaft.

32 Entsprechend diesem Forschungsstand wird die Mechanik Leonardos in den neueren Unternehmungen des Max-Planck-Instituts für Wissenschaftsgeschichte (MPIWG) zur Geschichte der Mechanik noch nicht behandelt. Die Handschriften Leonardos erscheinen weder in der schon sehr beachtlichen Internet-Präsentation des „Archimedes Project“ (http:// dmd.mpiwg-berlin.mpg.de/intro), noch in der zugehörigen Database Machine-Drawings von Wolfgang Lefèvre und Marcus Popplow (vgl. dies., ,,database machine drawings“. Ein Instrument zur Erschließung und Erforschung der Maschinenzeichnungen der RenaissanceIngenieure, in: Technikgeschichte 73, 2006, S. 115-120). Von großem Interesse für unser Projekt sind im Rahmen des ,Archimedes Project' dagegen die Maschinenzeichnungen des Francesco di Giorgio Martini, einschließlich der beigegebenen italienischen Texte und Zeichnungen, ebenso die Werke späterer Autoren. - Eine Wiedergabe der 1974 erschienenen Faksimile-Edition von Leonardos Codex Madrid I im Internet findet man im Angebot der Cornell University, New York, unter den so genannten KMODDL-eBooks (nur Faksimile), s. http://digital.library.cornell.edu/k/kmoddl/toc_leonardo1.html [Stand: 1.4.2009]. Den Gesamtbestand der Handschriften Leonardos im Internet-Überblick dokumentiert das Istituto di storia della scienza in Florenz unter Esposizioni e Didattica, Leonardo e gli ingegneri del Rinascimento (http://www.brunelleschi. imss.fl.it/genindice.asp.?appl=LIR\&Indice=63xsl= listagenerale\&chiave $=100775$. Es handelt sich um eine pädagogische Auswahlpräsentation nicht um eine Einzelaufbereitung mit genaueren technischen und historischen Erläuterungen.

33 Anregende, wenn auch sehr knappe Bemerkungen zum Codex Madrid I bei Stefan Klein, Da Vincis Vermächtnis oder: Wie Leonardo die Welt neu erfand, Frankfurt 2008, S. 165f. 
deshalb beispielhaft anhand von fol. $157^{\mathrm{v}}$ einige Ziele einer Neuedition des Codex Madrid I gegenüber der Edition von 1974 veranschaulicht werden.

\section{Codex Madrid, Band I, Faksimile fol. $157^{v}$ ohne Skizzenzählung}

Die Faksimileedition Retis von 1974 bietet keine Zählung der Einzelskizzen. Sie erschwert dadurch auf inhaltsreichen Seiten wie der vorliegenden die Zuordnung der Beischriften zu den Zeichnungen, die ohnehin schwierig bzw. zeitaufwändig ist, da die spiegelbildliche Schrift des Linkshänders Leonardo nicht ohne weiteres lesbar ist. Von dieser Edition gebe ich hier das Faksimile von fol. $157^{\mathrm{v}}$ aus Band I und eine fotografische Wiedergabe der Übersetzung aus Band IV der deutschen Ausgabe.

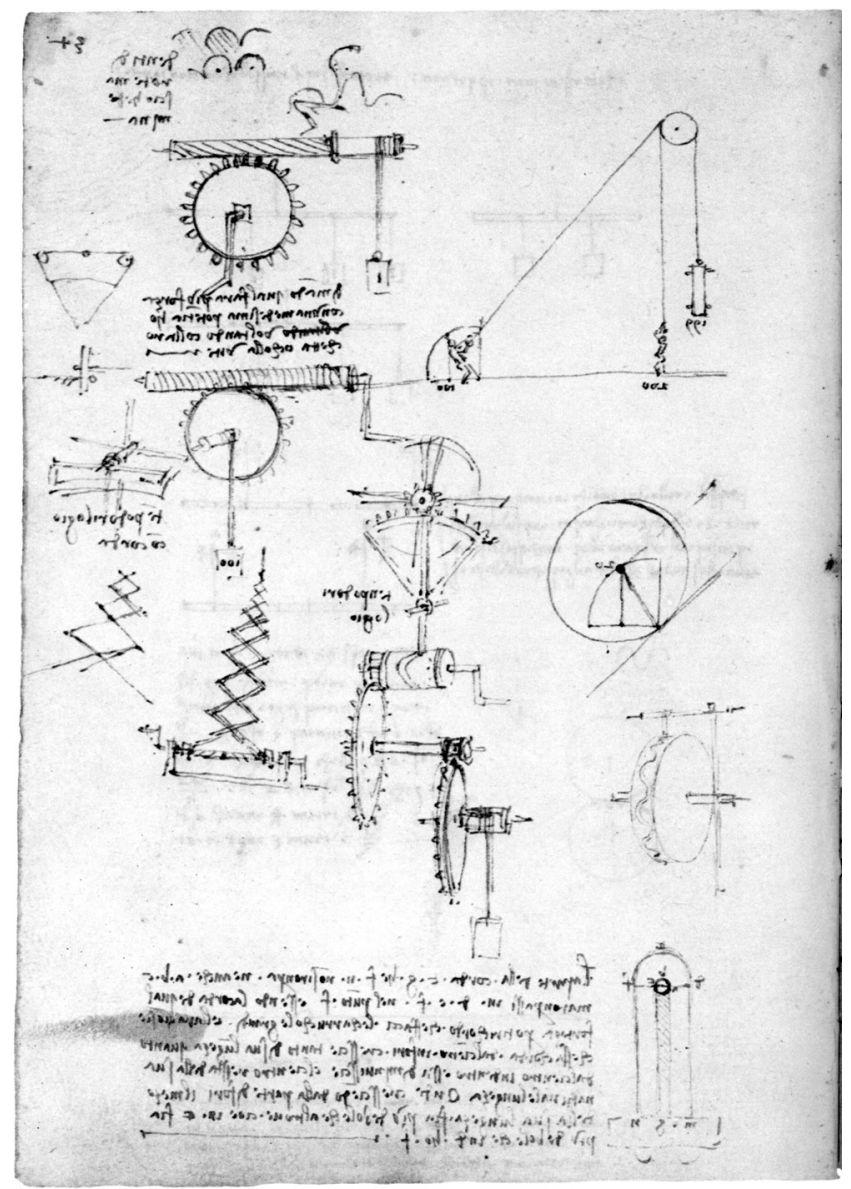

Hier sind S. 155ff. auch die Nachbauten von Mark Rosheim berücksichtigt, die inzwischen als selbständige Publikation erschienen sind, zum Verständnis von Codex Madrid I aber auch nur Details beisteuern, s. Mark Rosheim, Leonardo's Lost Robots, Berlin 2006; vgl. auch Domenico Laurenza, Mario Taddei u. Edoardo Zanon, Leonardo dreidimensional. Mit Computergrafik auf der Spur des genialen Erfinders, München 2006. 


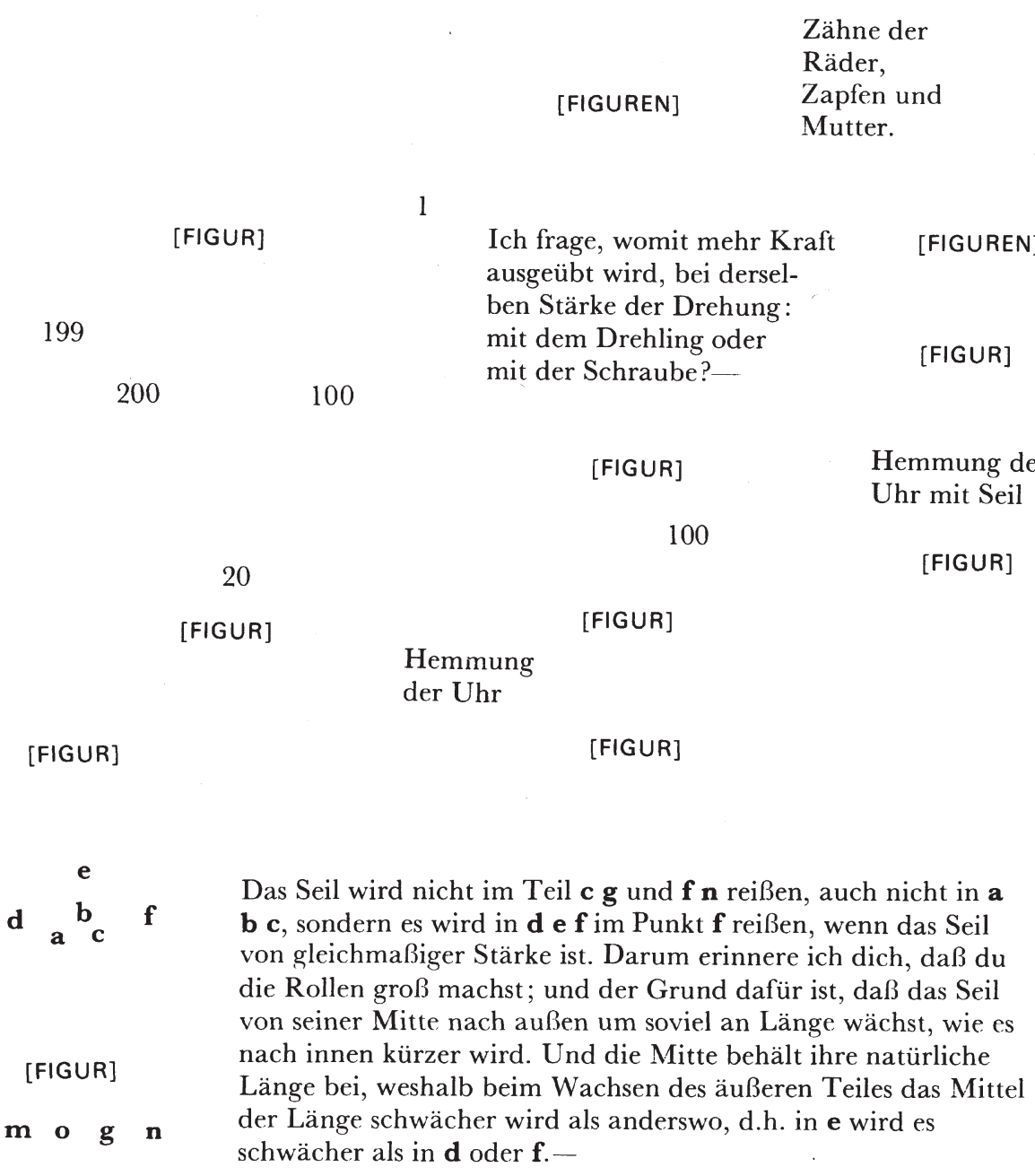

Technikgeschichte Bd. 76 (2009) H. 2 


\section{Neuedition}

Die Neuedition zeigt die Disposition Leonardos spiegelbildlich. Dadurch werden die Einträge identifizierbar und die rasche Zuordnung der Beischriften zu den Zeichnungen erleichtert. Zusätzlich erhalten die Einzelskizzen eine Zählung, die auf noch reicher angefüllten Seiten auch weit über 12 hinausgehen kann. Diese Zählung der Skizzen erfolgt in Entsprechung zu den Beischriften und zur inhaltlichen Struktur der Seite. Sie bereitet die sachliche Gliederung der Textedition und des Kommentars vor.

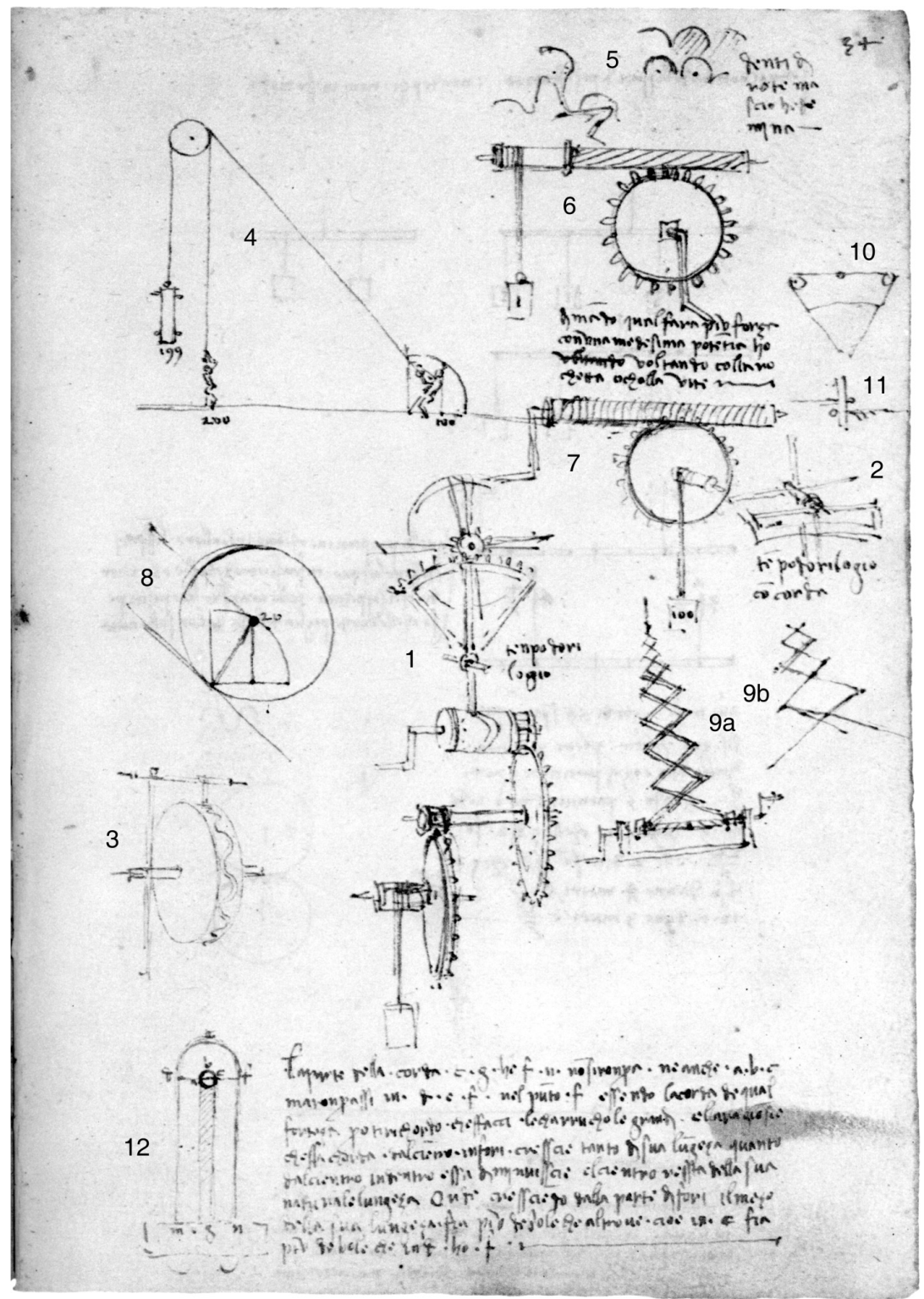




\section{A. Uhrenmechanik}

1) Uhrhemmung: Zahnräder und Ritzel, von einem Gewicht am Seil angetrieben; Hemmung durch Windflügel über einen Viertelzahnkranz, dessen Achse in der Sinus-Nut der mittleren Walze geführt wird

2) Windflügelhemmung einer Gewichtsuhr

3) Rad mit Sinus-Nut, darin geführt ein Stabpendel

1) tenpo d' orilogio

2) tenpo d' orilogio con corda.
Uhrhemmung

Uhrhemmung mit Seil

Kommentar zu A:

Fol. 157v dient als Beispiel für den häufig komplizierten Aufbau der Seiten im Codex Madrid I und die Schwierigkeit, klare Bezüge zwischen den Einzelskizzen herzustellen, die unbedingt eine Zählung erfordern. Die Wiedergabe erfolgt seitenverkehrt, um die Schrift lesbar zu machen. Zentrales Element ist die Uhrhemmung (fig. 1). Von den weiteren Apparaturen lassen sich aufgrund der Beischriften nur fig. 2-3 mit Sicherheit der Uhrhemmung zuordnen, doch ist nicht auszuschließen, dass Leonardo einen Zusammenhang zu den verschiedenen Möglichkeiten der Kraftübertragung in Abschnitt B gesehen hat. Zur Deutung von Abschnitt A ist vor allem Madrid I fol. 8r wichtig. Dort heißt es zu einer ähnlichen Skizze mit SinusNut wie hier fig. 3: „Bewegung, die wie eine Schwinge (rosta) hin und her geht und für die Hemmung der Uhren dient, da sie schwer (grave, 1974 „beständig") ist. Und das geschieht ohne Geräusch". Wie fig. 1 oben, zeigt auch fol. 8 fig. 5 eine Flügel- oder Windhemmung. Deren Erfindung gehört offenbar ins 15. Jh. Dondi um 1360 kennt sie noch nicht.

Bedini/Reti 1974, S. 258, nutzen fol. 157 fig. 3 und fol. 8 fig. 1 zur Untersuchung der Frage, wann zum ersten Mal als Regulator in Uhrwerken das Pendel verwendet wurde. Gewöhnlich verweist man dazu auf das Patent des Christian Huygens von 1657, doch zeigt sich die Verbindung von Pendel, Kronrad und Spindelhemmung auch schon 1525 in einer Handschrift der Uhrenbauerfamilie Volpaia in einer venezianischen Handschrift; der Florentiner Lorenzo della Volpaia war ein Freund Leonardos gewesen. Zu fig. 3 speziell notieren Bedi$\mathrm{ni} /$ Reti S. 259: „Der Anhänger ist verbunden mit der Stange eines Dinges, das ein Pendel zu sein scheint". Auf fol 8r fig. 1 ist das Pendel eindeutig. Leonardo erkannte andererseits den Isochronismus als grundlegende Eigenschaft des Pendels noch nicht, er betrachtete es zumindest nicht als grundsätzlich besseren Regulator im Vergleich zum Waagbalken. Verwiesen wird für die weitere Entwicklung auf die sinusförmige Führung in einer elektrischen Pendeluhr von Horstmann-Clifford und bei Zeitzündern im Zweiten Weltkrieg. Ob ein Zusammenhang zwischen der mechanischen Sinuskurve und der Entwicklung der Trigonometrie im späten Mittelalter besteht, bleibt offen.

\section{B. Kraftübertragungen}

4) Gewicht am Seil, gezogen über eine Umlenkrolle. Ein Mann zieht senkrecht, der andere im Winkel von ca. $45^{\circ}$

5) Runde Zähne von Zahnrädern und ihre konkaven Gegenstücke

6) Schnecke mit angehängtem Seil, angetrieben durch Zahnrad mit Kurbel

7) Zahnrad mit angehängtem Seil, angetrieben durch Schnecke mit Kurbel

8) Kreis auf schiefer Ebene mit eingefügtem Viertelkreis (?) 
9) a) Storchschnabel, ausgefahren von einer Doppelschnecke; b) Schema des Storchschnabels

10-11)?

5) denti di rote maschio e ffemina.

6) dimando qual farà più forza con una medesima potentia: o voltando colla rochetta o colla vite.
Zähne von Rädern, Zapfen und Mutter.

Ich frage, womit bei derselben Drehstärke mehr Kraft ausgeübt wird, mit dem Drehling oder mit der Schraube?

Kommentar zu B:

Fig. 4: Vgl. Codex Forster II, fol.73v mit erklärendem Text Leonardos (Uccelli S. 498). Im rechten Winkel sei die Leistung doppelt so hoch wie bei schrägem Zug.

Fig. 5: Sinn der Zahnradskizze an dieser Stelle noch ungeklärt.

Fig. 6-7: Vgl. Codex Atlanticus, fol. 1112r (400r), Kraftübertragung von Schnecke auf Kammrad. Zahnradtechnik Leonardos allgemein: Maschat 1989, S. 104-125.

Fig. 8: Sinn der geometrischen Konstruktion noch zu ermitteln.

Fig. 9: Feldhaus 1914, Sp. 766, kennt den Storchschnabel oder Pantographen erst als Erfindung des Jesuiten Christoph Scheiner zur Vergrößerung bzw. Verkleinerung von Zeichnungen. Eine Vorform finde sich bereits bei Heron von Alexandrien. Im Maschinenbau fand er, wie es scheint, schon im 13. Jh. Verwendung, beim Vorschub von Baumstämmen in Sägemühlen (Bechmann, Villard de Honnecourt, Paris 1991, S. 279f.).

\section{Zugkräfte auf innere und äußere Fäden eines gebogenen Seils}

\section{2) Dickes Seil, über eine kleine Rolle gelegt und an den Enden beschwert}

La parte della corda c-g e f-n non si ronperà, neanche $\mathbf{a}-\mathbf{b}-\mathbf{c}$, ma ronperassi in d-e-f nel punto $\mathbf{f}$, essendo la corda $\mathrm{d}^{\prime}$ equal forteza. Però ti ricordo che ffacci le carrucole grandi e lla ragion si è ch' essa corda dal cientro in fori cresscie tanto di sua lungheza quanto dal cientro in dentro essa diminuisscie. El cientro ressta della sua naturale lungheza, onde cressciendo dalla parte di fori, il mezo della sua lungheza fia più debole che altrove, cioè in $\mathbf{e}$ fia più debole che in $\mathbf{d}$ o $\mathbf{f}$.
Das Seil wird nicht im Teil c-g und f-n reiBen, auch nicht in $\mathbf{a}-\mathbf{b}-\mathbf{c}$, sondern es wird reißen in d-e-f im Punkt $f$, wenn das Seil gleichmäßig stark ist. Darum erinnere ich dich, dass du die Rollen groß machst; und der Grund dafür ist, dass das Seil von seiner Mitte nach außen um soviel an Länge wächst, wie es nach innen kürzer wird. Die Mitte behält ihre natürliche Länge bei, weshalb beim Wachsen des äußeren Teiles das Mittel der Länge schwächer wird als anderswo, das heißt in e wird es schwächer als in $\mathbf{d}$ oder $\mathbf{f}$.

\section{Kommentar zu C:}

Zur unterschiedlichen Verteilung der Zugkräfte auf die inneren und äußeren Fäden eines gebogenen Seils vgl. fol.154v, 155r-b, 157v-c, 164v, 165r des Codex Madrid I. Weiteres bei Maschat 1989, S. 170-172.

Anschrift des Verfassers: Prof. Dr. Dietrich Lohrmann, Historisches Institut, Theaterstr. 14, 52056 Aachen, E-Mail: Lohrmann@rwth-aachen.de 